\title{
Pressure-induced structural phase transitions in zirconia under high pressure
}

\author{
J. M. Leger, P. E. Tomaszewski, ${ }^{*}$ A. Atouf, and A. S. Pereira ${ }^{\dagger}$ \\ Laboratoire de Physico-Chimie des Materiaux, 1, pl. Aristide Briand, 92195 Meudon, France
}

(Received 15 December 1992)

\begin{abstract}
Angular-dispersive $\mathrm{x}$-ray in situ powder-diffraction experiments have been performed on pure zirconia, $\mathrm{ZrO}_{2}$, at room temperature under high pressure up to $50 \mathrm{GPa}$. Under increasing pressure four phases were successively encountered: baddeleyite (monoclinic, $P 2_{1} / c$ ) from normal pressure up to about $10 \mathrm{GPa}$, orthorhombic-I (Pbca) to $25 \mathrm{GPa}$, orthorhombic-II to $42 \mathrm{GPa}$, and orthorhombic-III above $42 \mathrm{GPa}$. The unit-cell parameters and the volume have been determined as a function of pressure. The bulk moduli of the two lower pressure phases have been calculated using Birch's equation of state. The bulk modulus of baddeleyite, $95 \mathrm{GPa}$, is much lower than expected from bulk modulus-volume systematics, $195 \mathrm{GPa}$, while for the orthorhombic-I phase, the experimental and calculated values are almost identical. A generalized $P$ - T diagram for $\mathrm{ZrO}_{2}$, including an orthorhombic-IV phase, is proposed and discussed. The phase transition to orthorhombic-II and orthorhombic-III phases can be described by a simple rotation of the unit cell of the orthorhombic-I phase about either the $b$ axis to form the orthorhombic-II phase or $a$ axis to form the orthorhombic-III phase. All high-pressure cells (orthorhombic-I, -II, and -III) have eight formula units $(Z=8)$. The orthorhombic-II phase was found not to have the cotunnite $\mathrm{PbCl}_{2}$-type structure which was proposed previously. There is no longer any example of a compound which transforms to such a cotunnite-type structure under high pressure. The behavior of zirconia and hafnia under high pressure is different although they have very close chemical properties at ambient pressure and identical structures in the two lower-pressure phases.
\end{abstract}

\section{INTRODUCTION}

Zirconia, $\mathrm{ZrO}_{2}$, is a major component of modern ceramic materials that exhibit very interesting mechanical properties when the high-temperature tetragonal phase has been partially stabilized to produce the socalled tough hardening phenomena. Thus the crystal structure of zirconia polymorphs and the mechanisms of transitions between them are of considerable interest in view of their connection with the properties of advanced zirconia-based ceramics.

The high-pressure, high-temperature phase diagram of $\mathrm{ZrO}_{2}$ has been extensively studied to determine the fields of stability of the different phases and bring some understanding to the mechanisms of the phase transitions, mainly the monoclinic-to-tetragonal one which is of martensitic nature. Moreover, the high-pressure behavior of zirconia can provide a useful analogy for the pressureinduced phase transitions in silica, which are important in relation to the study of the deep interior parts of the Earth.

The lack of reliable structural data on the highpressure phases other than orthorhombic-I phase (with a correct space group of $\mathrm{Pbca}$ ), motivated us to perform in situ measurements of the lattice parameters and determine the equations of state for the phases of $\mathrm{ZrO}_{2}$ existing at room temperature. Thus, we have performed powder x-ray-diffraction experiments up to $50 \mathrm{GPa}(500$ kbar) on pure zirconia. Before proceeding to the description of the experimental procedure and results, it is useful to summarize some data about the structural properties.

The numerous data on pure and stabilized zirconia, which can be found in the literature, should be taken, however, while paying special attention to several shortcomings or/and difficulties in the obtainment of meaningful experimental data and their correct interpretation. Therefore, we note the following.

(a) The use of $x$ rays instead of neutrons, produce difficulties in the correct interpretation of the diffraction patterns of $\mathrm{ZrO}_{2}$ due to small scattering factor from oxygen atoms (the $\mathrm{x}$-ray-diffraction pattern could then be indexed based on an incorrect unit-cell symmetry as different positions of oxygen atoms might be indistinguishable). Attention should therefore be focused on neutron data.

(b) The diffraction pattern can be representative either of the bulk, as in x-ray or neutron diffraction, or the surface as in electron microscopy.

(c) The use of doped sample, e.g., stabilized $\mathrm{ZrO}_{2}$ ceramics, can result in data which cannot be compared to data obtained from pure zirconia.

(d) The use of powdered samples does not provide direct information about the symmetry of the crystal studied.

(e) Measurements made on quenched samples far from thermodynamic equilibrium and without knowledge of the real history of the treated sample can result in an incorrect interpretation of the data (e.g., the case of a multiphases mixture).

(f) Conditions of the in situ measurements under pressure are extremely difficult.

Accordingly, the full crystal structure data where it exists should be considered first. The data on the lattice parameters alone or even with a more or less strictly determined space group, can result in an incorrect structure for the high-pressure phases. 


\section{A. Phase diagram of zirconia}

Considering the conditions and restrictions on the interpretation of data mentioned above, we suggest the generalized pressure-temperature phase diagram for zirconia based on the literature ${ }^{1-23}$ in addition to this work.

Recent research has revealed the existence of several, at least seven, polymorphs of $\mathrm{ZrO}_{2}$ which occur in different ranges of temperature and pressure. They are presented in Fig. 1. The stability fields of the different phases were determined either by in situ detection of phase transitions or by searching for different phases in samples quenched from different $P$ - $T$ conditions.

At ambient pressure, the crystal structures of $\mathrm{ZrO}_{2}$ polymorphs (monoclinic, tetragonal, and cubic) were studied using neutron diffraction with considerable precision up to the highest temperatures. ${ }^{19,23-27}$ However, there are some controversies about the existence of an intermediate orthorhombic phase observed on heating only, and a possible second high-temperature tetragonal phase. ${ }^{19,21,28}$ Some crystal structure data are presented in Table I. The cubic $\mathrm{ZrO}_{2}$ phase has the fluorite structure. Most of the other polymorphs, especially tetragonal and monoclinic, are derivatives of this structure. In a general description, these structures consist of layers of $\mathrm{Zr}$ atoms between two oxygen layers as in the amorphous form. ${ }^{29,30}$ Otherwise speaking, the structures are built from the fluorite structure by slight adjustments of the unit-cell parameters and by displacing oxygen atoms alternating above and below their ideal position in the $(010)$ plane in cubic phase. ${ }^{21,31}$

\section{B. High-pressure phases}

At higher pressure the situation is more complex as there are examples of contradictory data in the literature. Now, it seem obvious that all the high-pressure phases existing below $50 \mathrm{GPa}$ and $1200 \mathrm{~K}$ are orthorhombic as is discussed below. However, only the structure of the high-pressure phase labeled as orthorhombic-I has been

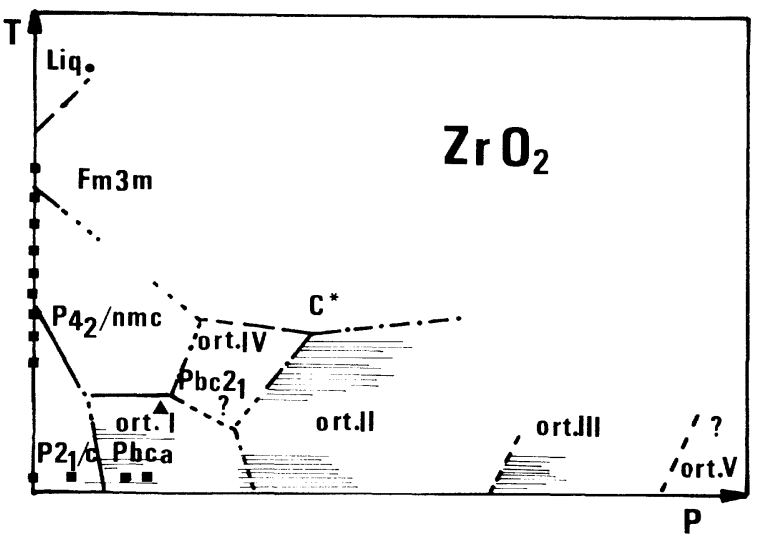

FIG. 1. Generalized pressure-temperature phase diagram for $\mathrm{ZrO}_{2}$. The stability fields of the different phases were determined either by in situ detection of phase transition (full lines in the diagram) or by search for different phases in samples quenched from different $P-T$ conditions (dotted-dashed lines). Dashed lines correspond to those obtained from calculations and dotted lines are from our propositions. The hatched regions indicate the coexistence of phases or the uncertainties and/or inconsistencies of the data in the literature. The squares mark the full structure analysis made in situ and the triangle on quenched samples.

obtained by in situ crystal structure analysis; ${ }^{11}$ two other structure refinements on this phase have been made on quenched samples. ${ }^{20,32}$ There are several other examples of orthorhombic symmetries for pure or partially stabilized $\mathrm{ZrO}_{2}$ in different conditions.

The most controversial issues were concerning the phase labeled as orthorhombic-I (Ortho-I) which was first considered to be of tetragonal symmetry, but different from that observed at high temperature. After several years of extensive structure studies on different samples, one can firmly state that the correct space group of the

TABLE I. Literature data on space groups and lattice parameters for room- and high-temperature phases of $\mathrm{ZrO}_{2}$ obtained by $\mathrm{x}$-ray $(X)$ or neutron $(N)$ diffraction. The reliability factor $R$ is also given for data from full structure determination on powder samples; only the data from Ref. 31 are from a single-crystal experiment ( $X$ mono).

\begin{tabular}{rllllllll}
\hline \hline$T(\mathrm{~K})$ & Space group & $a(\AA)$ & $b(\AA)$ & $c(\AA)$ & $\beta(\mathrm{deg})$ & $N / X$ & $R(\%)$ & Ref. \\
\hline \multirow{2}{*}{690} & $F m 3 m$ & 5.230 & & & & $X$ & & $\mathrm{a}$ \\
& & 5.269 & & & & $N$ & & $\mathrm{~b}$ \\
2440 & $P 4_{2} / n m c$ & 5.2022 & & 5.3175 & & & & \\
1900 & & 5.1727 & & 5.3048 & & $N$ & 8.6 & $\mathrm{c}$ \\
1630 & & 5.1458 & & 5.2864 & & $N$ & 1.6 & $\mathrm{~b}$ \\
1100 & & 5.1239 & & 5.2413 & & $N$ & 6.45 & $\mathrm{c}$ \\
& & & & & & & \\
1100 & $P 2_{1} / c$ & 5.1828 & 5.2117 & 5.3731 & 98.835 & $N$ & 6.45 & $\mathrm{c}$ \\
300 & & 5.1495 & 5.2021 & 5.3198 & 99.238 & $N$ & 8.35 & $\mathrm{c}$ \\
300 & & 5.145 & 5.2075 & 5.3107 & 99.233 & $X$ mono & 9.0 & $\mathrm{~d}$ \\
\hline \hline
\end{tabular}

${ }^{a}$ Reference 21.

${ }^{\mathrm{b}}$ Reference 26.

${ }^{\mathrm{c}}$ Reference 23 .

${ }^{\mathrm{d}}$ Reference 31 . 
Ortho-I phase is Pbca (No. 61) with the unit-cell parameters of about 10-5-5 $\AA$, respectively (twice $a$ parameter with respect to the $P b c m$ (No. 57) disordered structure). ${ }^{20}$ Selected data for this phase are presented in Table II. The crystal with the Pbca structure consists of a planar periodical arrangement of alternate obverse and reverse domains (more precisely: alternate types of an O1-atom net), leading to a doubling of the unit-cell parameter $a .^{33}$ These domains have a $P b c 2_{1}$ (No. 29) symmetry and only one type of $\mathrm{O} 1$-atom net ${ }^{34}$ is displaced from its position in the tetragonal high-temperature phase. Thus, this phase can be regarded as intermediate between the tretragonal and the monoclinic ones. ${ }^{35}$ The average of domains of noncentrosymmetric $P b c 2_{1}$ symmetry yields the centrosymmetric $\mathrm{Pbcm}$ (No. 57) structure model known from $\mathrm{x}$-ray-diffraction data ${ }^{11,32}$ and not confirmed by neutron experiments. ${ }^{33}$ In this structure the oxygen $\mathrm{O}(1)$ is disordered over the two sets of positions related by the mirror plane at $z=\frac{1}{4}$. It should be noticed that the coordination number does not change with respect to that in the monoclinic phase: It remains equal to seven. The proposed value of eight (Ref. 7) was based on the tetragonal high-pressure structure, which has been shown to be wrong.

The existence of the phase labeled as orthorhombic-II (Ortho-II) was determined by several studies. From quenching experiments the symmetry was assigned as Pnma (No. 62) and the cotunnite, $\mathrm{PbCl}_{2}$-type, structure was proposed. ${ }^{7}$ Some verification by powder refinement was reported on $\mathrm{CaO}$-stabilized zirconia ${ }^{36}$ but no details were given. The present experiment data on pure $\mathrm{ZrO}_{2}$ under high pressure do not confirm this model.

Discussion of the orthorhombic-III (Ortho-III) phase is also included in this paper. Here it should be pointed out that the discovery of this phase above $35 \mathrm{GPa}$ was previously discussed in terms of a tetragonal symmetry. ${ }^{18}$
The phase boundaries obtained from the different experiments and those added based on thermodynamic criteria provide evidence for the existence of a $P-T$ field for a new phase labeled here as orthorhombic-IV (Ortho-IV). Actually, quenching experiments from these $P-T$ conditions indicated an orthorhombic symmetry for this phase; however, the exact symmetry was not directly proved under high-pressure, high-temperature conditions. Both space groups proposed, $P 2_{1} 2_{1} 2_{1}$ (No. 19) (Ref. 3) and Pbca (No. 61) (Ref. 20), should be rejected: the first one was already rejected because of the too few Raman lines observed under pressure ${ }^{8}$ and the second corresponds to the Ortho-I phase. A possible solution within orthorhombic symmetry is to propose the space group $P b c 2_{1}$ (No 29) as was already found in electron-diffraction experiments for thin-foil specimens of stabilized zirconia $^{37-41}$ and known as the symmetry of the domains which form the Ortho-I phase. ${ }^{34}$

There are also some indications of a further phase transition at pressures higher than $55 \mathrm{GPa}$ to a phase of a symmetry which is probably higher than orthorhombic. ${ }^{16}$ [The proposed cubic symmetry may be due, in our opinion, to a misinterpretation of the $\mathrm{x}$-ray-diffraction lines which come mostly from the zirconium framework. Actually, if the framework of the oxygen atoms is also considered, then the highest possible symmetry should be orthorhombic Pmmm (No. 47) in order to retain the same structural motif. The lattice parameters would then be of about $2.5 \AA$ with the oxygen atoms in the $1(a)$ and $1(d)$ positions, while $\mathrm{Zr}$ atoms occupy $2(r)$ positions with a probability of 0.5 .

In the high-temperature, high-pressure region of the $P-T$ diagram, the phase labeled as $C^{*}$ (Ref. 17) seems to be the same as the well-known high-temperature, ambient-pressure cubic phase, but no experimental confirmation of this has been performed.

TABLE II. Selected data for high-pressure Ortho-I phase of $\mathrm{ZrO}_{2}$ (only Ref. 34 concerns a partially stabilized zirconia) from $\mathrm{x}$-ray $(X)$ and neutron $(N)$ experiments made in situ $(s)$ or on quenched samples $(q)$. Pressure and temperature conditions are given in $[\mathrm{GPa}]$ or $[\mathrm{K}]$ units, respectively, (AP = ambient pressure, $\mathbf{R T}=$ room temperature $)$.

\begin{tabular}{|c|c|c|c|c|c|c|c|c|}
\hline Symm. & $a(\AA)$ & $b \AA$ ) & $c(\AA)$ & $V\left(\AA^{3}\right)$ & $R_{B}(\%)$ & $X / N$ & $P ; T$ & Ref. \\
\hline$P 2{ }_{1}{ }_{1}{ }_{1}$ & 5.036 & 5.267 & 5.073 & 134.6 & & $X$ & $4.0 ; 2070 q$ & a \\
\hline$P 2_{1} 2_{1} 2_{1}$ & 5.016 & 5.230 & 5.016 & 131.6 & & $X$ & $9.9 ; 730 s$ & $\mathrm{~b}$ \\
\hline Tetr. & 5.009 & 5.237 & 5.009 & 131.4 & & $X$ & 8.3;RTs & c \\
\hline Tetr. & 5.046 & 5.129 & 5.046 & 130.6 & & $X$ & $7.3 ; 1270 q$ & d \\
\hline Ortho. & 5.042 & 5.257 & 5.092 & 135.0 & & $X$ & $6.0 ; 870 q$ & $\mathrm{e}$ \\
\hline Pbcm & 5.005 & 5.235 & 5.051 & 132.3 & 3.4 & $X$ & 3.9;RTs & $f$ \\
\hline Pbcm & 4.992 & 5.229 & 5.046 & 131.7 & 4.5 & $X$ & 5.1;RTs & f \\
\hline Pbcm & 5.036 & 5.255 & 5.086 & 134.6 & 5.0 & $X$ & $6.0 ; 870 q$ & $\mathrm{~g}$ \\
\hline$P b c 2_{1}$ & 5.068 & 5.260 & 5.077 & 135.3 & 2.1 & $N$ & $\mathrm{AP} ; 30 q$ & h \\
\hline$P b c a$ & 10.086 & 5.262 & 5.091 & 270.2 & 3.9 & $N$ & $6.0 ; 870 q$ & $\mathrm{i}$ \\
\hline Ortho. & 5.047 & 5.303 & 5.207 & 139.4 & & $X$ & AP; $1370 s$ & $\mathrm{j}$ \\
\hline
\end{tabular}

${ }^{a}$ Reference 3.

${ }^{b}$ Reference 6.

${ }^{\mathrm{c}}$ Reference 9.

${ }^{\mathrm{d}}$ Reference 10 .

${ }^{\mathrm{e}}$ Reference 56.
${ }^{\mathrm{f}}$ Reference 11.

${ }^{\mathrm{g}}$ Reference 32.

${ }^{\mathrm{h}}$ Reference 34 .

iReference 20.

${ }^{\mathrm{j}}$ Reference 21. 


\section{EXPERIMENTAL PROCEDURE}

The powder $x$-ray-diffraction experiments were performed at room temperature using a diamond-anvil cell of the lever-arm type with diamonds of $500-\mu$ m diameter flats. The zirconium oxide was a powder with a grain size below $1 \mu \mathrm{m}$ and a stated purity better than $99.9 \%$. The 4:1 methanol-ethanol mixture generally used as pressure transmitting medium introduces some discontinuities in the resulting data ${ }^{42}$ when it solidifies and becomes glassy at room temperature at around 10-12 GPa, so silicon oil was preferred. The mixture of silicon oil and sample was loaded in the $150-\mu \mathrm{m}$ diameter hole drilled in a T301 gasket of initial thickness $250 \mu \mathrm{m}$, preintended to $100 \mu \mathrm{m}$. Some additional experiments were performed below $10 \mathrm{GPa}$ with the 16:3:1 methanol-ethanol-water mixture to further study the monoclinic-orthorhombic-I phase transition. A single-crystal ruby chip was placed on the upper surface of the sample as the pressure calibrant. The value of the pressure was calculated from the fifth power of the wavelength shift of the $R_{1}$ fluorescence line (fit with an anisotropic stress component) although the two maxima of the doublet were always clearly seen. The powder $\mathrm{x}$-ray-diffraction patterns were recorded on wet films using the molybdenum radiation from a fine focus tube. The filtering of the $K \beta$ radiation was made less effective than usual to shorten the exposures to about $24 \mathrm{~h}$. A few additional experiments were performed with increased filtering to check for the possible presence of lines due to the $K \beta$ radiation. A wide slit in the seat of the rear diamond allowed for recording of the diffraction patterns up to about $2 \theta= \pm 40^{\circ}$ on films placed on a cylin-

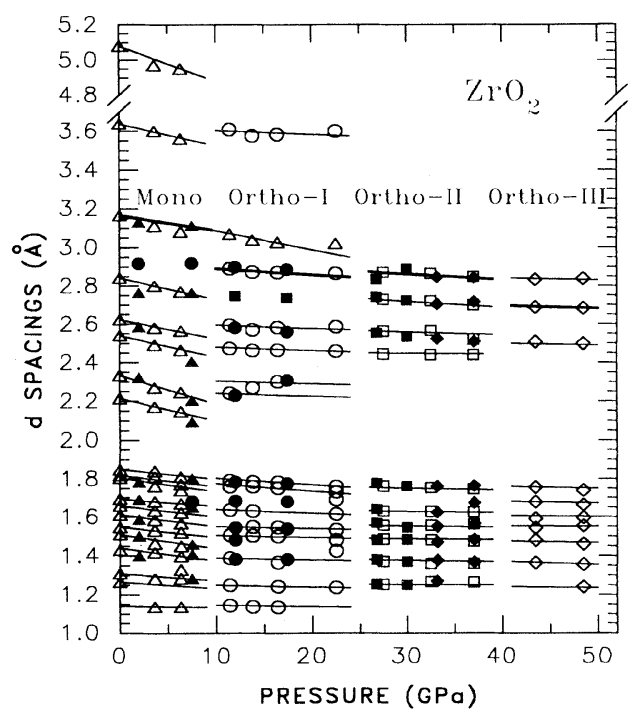

FIG. 2. Pressure dependence of the $d$ spacings $d$ of $\mathrm{ZrO}_{2}$. Open symbols: increasing pressure, filled symbols: decreasing pressure; $\triangle$ : monoclinic phase; $\bigcirc$ : orthorhombic-I phase; $\square$ : orthorhombic-II phase, and $\diamond$ : orthorhombic-III phase. The lines are there to guide the eye (for increasing pressure only); the thick line is for the highest intensity line in each phase. The diffraction lines which possibly coincided with the lines from gasket (mainly iron) were omitted in the figure. drical support, with a sample-to-film distance of 25.47 $\mathrm{mm} .{ }^{42}$ The diameters of the Deybe rings were measured with a low magnification microscope. The diffraction lines, which possibly coincided with the lines from gasket (mainly iron), were omitted in the discussion of our data. Indexing of the powder diagrams was performed using the DICVOL program. ${ }^{43}$ Only single diffraction lines were used for lattice parameters refinements.

The experimental data on the pressure dependence of the relative volume $V / V_{0}$ obtained with the increasing pressure were fitted to Birch's equation of state: ${ }^{44}$

$$
P=\left(\frac{3}{2}\right) B_{0} x(1+x)^{5 / 2}(1+a x),
$$

where $x=\left(V / V_{0}\right)^{-2 / 3}-1$ and $a=(3 / 4)\left(B_{0}^{\prime}-4\right) . \quad B$ is the bulk modulus and $B^{\prime}$ its first pressure derivative; the subscript zero refers to values at normal pressure.

After pressurization to $47.5 \mathrm{GPa}$, additional diffraction patterns were recorded when the pressure in the diamond-anvil cell was gradually decreased.

\section{RESULTS AND DISCUSSION}

All experimental data, i.e., $d$ spacings calculated from the positions of diffraction lines on the x-ray films, lattice parameters, and relative volume, are plotted vs pressure in Figs. 2, 3, and 4, respectively; in addition, representative data for each phase are presented in Table III. Four pressure regions are clearly seen which correspond to four different phases. Upon decreasing the pressure, an identical sequence of phase transitions was observed. All phase transitions were fully reversible and only some hysteresis was found. We could retain neither the orthorhombic-III phase down to normal pressure, con-

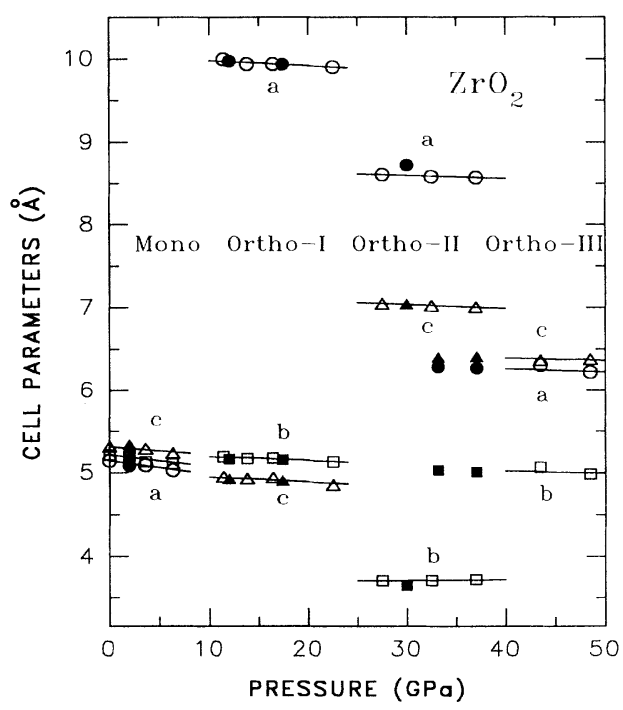

FIG. 3. Unit-cell parameters of $\mathrm{ZrO}_{2}$ as a function of pressure. $\bigcirc$ : a parameter; $\square$ : b parameter; $\triangle$ : c parameter (open symbols: increasing pressure, filled symbols: decreasing pressure). With this choice of parameters, the structural motif can be conserved through all the crystallographic transitions. The lines are there to guide the eye. The monoclinic angle $\left(99.8^{\circ}\right)$ of the monoclinic phase does not vary significantly. 
trary to the data reported in Refs. 18 and 45, perhaps due to the gradual decrease of the pressure in our experiments.

\section{A. The baddeleyite monoclinic phase}

Twenty one diffraction lines were observed in our experiments under pressure. The strongest line 111 was still observed at $22 \mathrm{GPa}$, while the other lines disappeared below about $10 \mathrm{GPa}$. The cell parameters are in good agreement with the values obtained at 3.9 and 5.1 GPa from single-crystal x-ray diffraction. ${ }^{11}$ The pressure variations of the unit-cell volume allowed us to calculate the bulk modulus: $B_{0}=95 \pm 8 \mathrm{GPa}$ for usual values, $4-5$, of the first pressure derivative of the bulk modulus (as the pressure range of stability of the monoclinic phase is narrow, the exact value of $B_{0}^{\prime}$ does not influence the value of the bulk modulus). This is smaller than a value of 135 $\mathrm{GPa}$ deduced from the shear and elasticity moduli of polycrystalline samples measured under normal conditions and extrapolated to zero porosity. ${ }^{46}$ Recent Brillouin scattering experiments ${ }^{47}$ at normal pressure performed on single crystals gave a mean value of $185 \mathrm{GPa}$, but these measurements could have been perturbed by

TABLE III. Interplanar distances (observed and calculated in first and second columns, respectively) with their indices and unitcell parameters for each phase of $\mathrm{ZrO}_{2}$ under high pressure ( $*$ : line of highest intensity; $m$ : line from the monoclinic phase;-: line observed under other pressure; $Z$ : formula units in the unit cell).

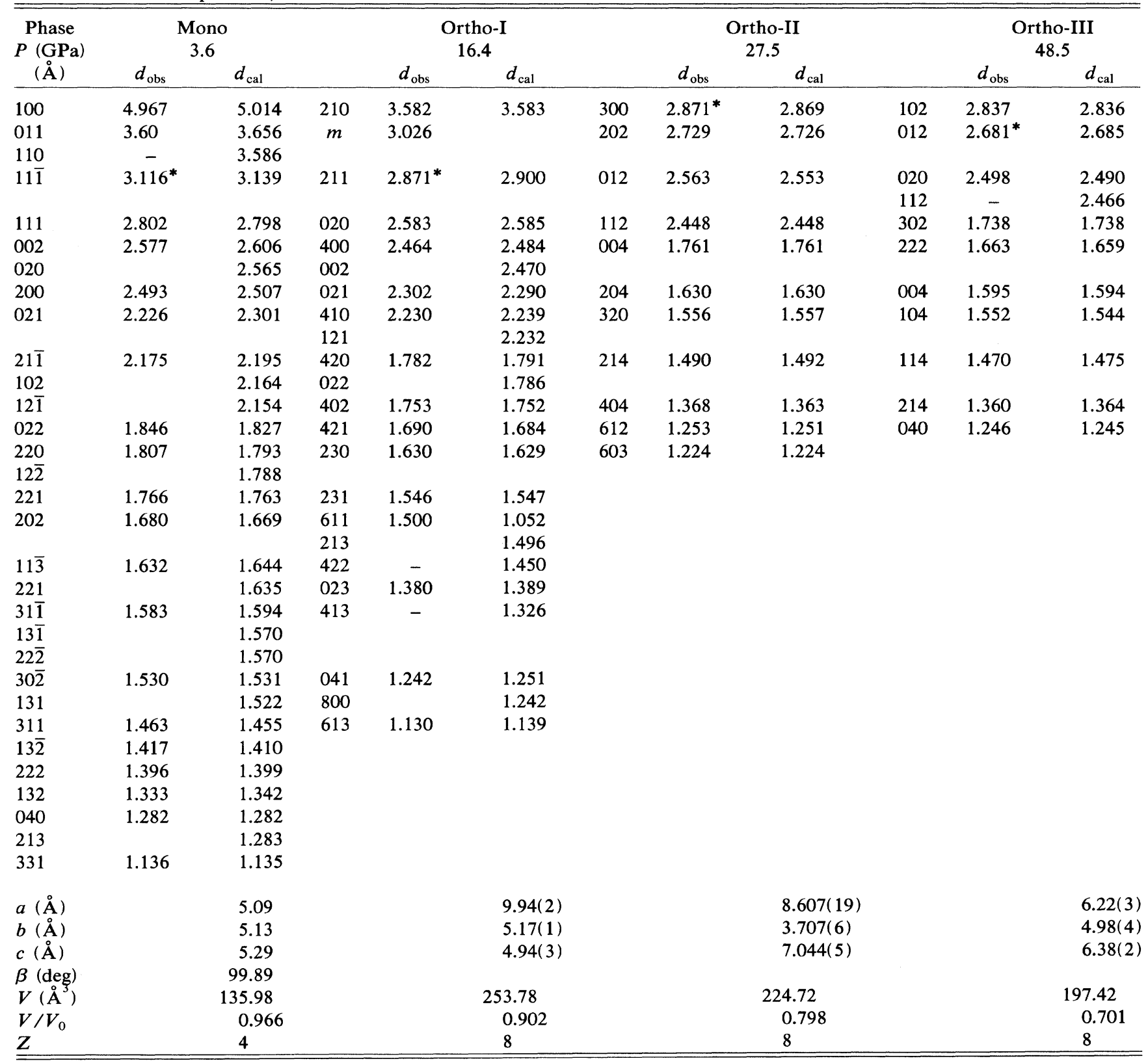




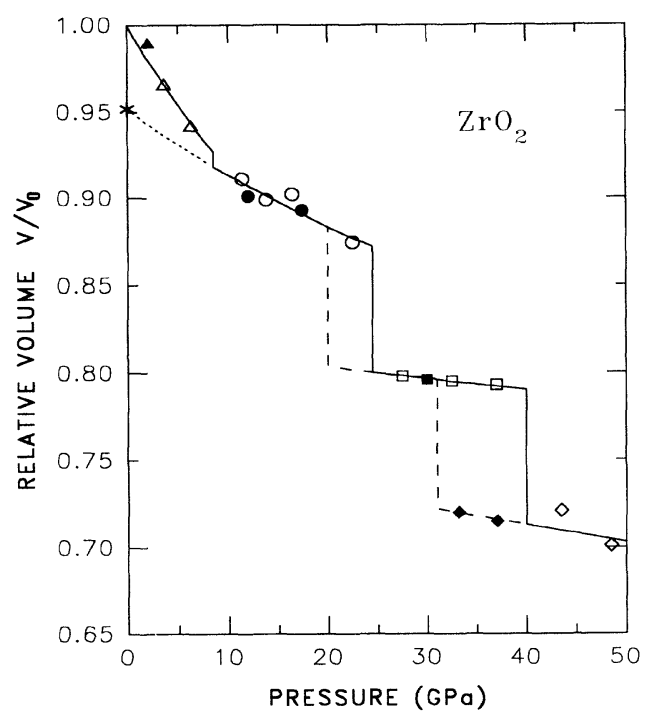

FIG. 4. Relative volume of $\mathrm{ZrO}_{2}$ as a function of pressure. The graphic symbols are the same as in Fig. 2. The lines are there to guide the eye; dashed line: decreasing pressure, dotted line: fit of the data of the orthorhombic-I phase to the Birch equation using the volume under ambient condition obtained from quenched samples $(20,34)$.

twinning as large crystals of the monoclinic phase are extremely difficult to make. From shock-wave data, the bulk modulus was inferred to be $149 \mathrm{GPa}{ }^{48}$

The experimental bulk modulus is much lower than expected (195 GPa) from bulk modulus-volume systematics:

$$
B_{0}[\mathrm{GPa}]=700 S^{2} Z_{A} Z_{C} / V_{0},
$$

where $V_{0}$ is the mean molar volume of an atomic pair, $S$ the ionicity ( $S^{2}=0.5$ for oxides), and $Z_{A}$ and $Z_{C}$ the formal charges of the anion and cation, respectively. ${ }^{49}$ The experimental polyhedral bulk modulus can be calculated from previous data. ${ }^{11}$ It is equal to $110 \mathrm{GPa}$ and close to the volume bulk modulus. This indicates that there is no polyhedral tilting, as expected for a structure which is only slightly deformed with respect to the parent fluorite structure. Furthermore, no increase of the distortion of the unit cell with pressure could be evidenced. This experimental value is much lower than the value, $300 \mathrm{GPa}$, deduced from the polyhedral bulk modulus - versus the cation-anion distance relationship: ${ }^{50}$

$$
B_{p}=750 S^{2} Z_{A} Z_{C} / d^{3},
$$

where $d(\AA)$ is the mean cation-anion distance. The lower experimental values for the volume bulk modulus and the polyhedral bulk modulus show that the monoclinic phase is possibly stabilized by symmetry-breaking defects. It could also arise from the partial softening of an acoustic mode occurring prior to the martensitic shear transformation. This is in good agreement with the results of the calculation within the potential-induced breathing model. Using this model, equations of state, relative phase stabilities, and elasticity of several different structures of $\mathrm{ZrO}_{2}$ were calculated. ${ }^{51}$ Interestingly enough, the monoclinic structure was found not to be the stable phase at ambient pressure. A monoclinic structure with a bulk modulus equal to $152 \mathrm{GPa}$ was found to be stable, but at lower density than under ambient conditions. At higher densities, orthorhombic phases of $\mathrm{ZrO}_{2}$ were found to be stable with bulk moduli of 273 and 314 $\mathrm{GPa}$, respectively.

\section{B. The orthorhombic-I phase}

Between 8 and $11 \mathrm{GPa}$ a phase transition was observed to take place in both silicon oil and liquid medium in general agreement with the literature data. Furthermore, shearing experiment on zirconia failed to detect this transition to $5 \mathrm{GPa}$. It is well known that this transition is sensitive to the grain size; in a single crystal it occurs at much lower pressure, $3.6 \mathrm{GPa}^{52}$ On releasing the pressure the most intense line can be seen down to $3 \mathrm{GPa}$.

Seventeen diffraction lines from this phase were observed. They could be indexed under orthorhombic symmetry and their intensities were in agreement with the proposed Pbca (No. 61) structure model. The bulk modulus of the orthorhombic-I phase was calculated using the volume $V /\left.V_{0}\right|_{p=0}=0.952$, the average value from the neutron ${ }^{2}$ and $x$-ray-diffraction data, ${ }^{34}$ both on a quenched sample. The first pressure derivative, $B_{0}^{\prime}$, was assumed to have the usual value of 5 . The resulting bulk modulus is about $220 \mathrm{GPa}$ and close to the expected one from the bulk modulus-volume relationship, $205 \mathrm{GPa}$. The volume change at the monoclinic to orthorhombic-I transition $(0.8 \%)$ was smaller than the value obtained from the single-crystal study under hydrostatic pressure $(3 \%) .{ }^{11}$ It is evidence that the large difference in bulk moduli for the two phases induces a reduction in the volume decrease when the transition is shifted to higher pressures, as in this case.

\section{The orthorhombic-II phase}

The phase transition to the orthorhombic-II phase occurred between 22 and $27 \mathrm{GPa}$. The transition pressure was higher than reported in Ref. 9, because it was also sluggish, as was the lower-pressure one. On releasing the pressure the diffraction lines of this phase appeared at about $32 \mathrm{GPa}$ and persisted down to $11 \mathrm{GPa}$ (in coexistence with the lines of orthorhombic-I phase below about $20 \mathrm{GPa}$ ).

All 11 diffraction lines could easily be indexed on an orthorhombic cell with lattice parameters of about 8.6, 3.7 , and $3.5 \AA$. The molar volume decreased by $7.1 \%$ at this transition.

The cotunnite, $\mathrm{PbCl}_{2}$-type, structure was proposed for this phase. ${ }^{7,36}$ However, the line at about $2.45 \AA$, which was observed in situ in our experiment and in Ref. 18, is forbidden in this structure. Thus, such structure model should be rejected. Furthermore, as far as the intensities of the diffraction lines are concerned, it is obvious that this line cannot be interpreted as the 002 line from the lower-pressure orthorhombic-I phase; the line 402 of similar intensity already vanished at about $18 \mathrm{GPa}$. 
Moreover, the pressure dependence of the position of the lines of highest intensity in both phases (211 for orthorhombic-I and 300 for orthorhombic-II) displayed a noticeable discontinuity at the transition indicating the absence of the orthorhombic-I phase for pressures higher than $25 \mathrm{GPa}$. Thus, our in situ measurements did not confirm the existence of $\mathrm{PbCl}_{2}$-type structure in $\mathrm{ZrO}_{2}$ at room temperature under high pressure.

There is still further evidence for rejecting the $\mathbf{P b C l}_{2}$ type structure as a structure model for the orthorhombic-II phase. At ambient pressure, although in Ref. 7 or in Ref. 36 there is no report of this line at about $2.45 \AA$ on quenched samples, the intensities of other $\mathrm{x}$ ray-diffraction lines are not in good agreement with the calculated values for a $\mathrm{PbCl}_{2}$-type structure. Mainly, one characteristic and intense line of the proposed cotunnite structure at $d=2.78 \AA\left(I / I_{0}=48\right)$ was not reported; under normal conditions it should be well separated from the line at $2.80 \AA\left(I / I_{0}=96\right)$. Moreover, it was not clear if additional lines (e.g., from the monoclinic phase) to the lines given in the paper $^{7}$ were excluded from the published data or not. In the last case, the reported diagram could be explained by a mixture of several phases: monoclinic, orthorhombic-I and -II, as is the case for $\mathbf{H f O}_{2}{ }^{53}$ Furthermore, the transition from the orthorhombic-I phase to a $\mathrm{PbCl}_{2}$-type phase could require a great mutual displacement of both, zirconium and oxygen, sublattices. After the transition, both type of atoms would lie in the same planes; this seems to be unreasonable and questionable.

The nonexistence of the $\mathrm{PbCl}_{2}$-type structure at high pressure, at least at room temperature, is of great importance because it leads to changes in the well-known diagram of the molar volume of $\mathrm{MO}_{2}$ compounds vs the third power of the $M$-O distance. ${ }^{45,54}$ The proposed cotunnite-type high-pressure phase has no instances: There are neither $\mathrm{ZrO}_{2}$ (this work) nor $\mathrm{HfO}_{2}$ (Ref. 53) phases of this structure type at room-temperature highpressure conditions.

It seems to be reasonable to retain the same general structure motif for zirconia in this phase. The similar conservation of the motif was observed in the phase transition from the mononclinic to orthorhombic-I phase. This led us to double the unit cell with respect to that found from our x-ray-diffraction data. Unfortunately, it is not possible to determine here which cell parameter, $b$ or $c$, should be doubled. This cell doubling is not possible to detect in our experiments because of the small scattering factor of the oxygen atoms. For simplicity of the description only, especially in Table III, we chose to double the $c$ axis. Within this model the phase transition from orthorhombic-I to orthorhombic-II phase is described as a rotation about the $a$ axis providing to the following relations between unit cells: $\mathbf{a}_{2}=\mathbf{a}_{1}$, $\mathbf{b}_{2}=(1 / 2)\left(\mathbf{b}_{1}+\mathbf{c}_{1}\right), \mathbf{c}_{2}=\mathbf{b}_{1}-\mathbf{c}_{1}$. The preliminary powder diagram simulation using such model gives satisfactory agreement between observed and calculated line intensities. Unfortunately, the deduction of possible space group(s) is not possible.

The bulk modulus and the initial volume of the orthorhombic-II phase could not be calculated reliably because this phase could not be retained down to ambient pressure in our experiment. Nor the value $\left(V /\left.V_{0}\right|_{P=0}\right.$ given in the literature ${ }^{7}$ could be used, as it corresponds to another unit cell and no value could be derived for the orthorhombic-II cell.

\section{The orthorhombic-III phase}

A third pressure-induced phase transition in zirconia occurred between 37.5 and $42.5 \mathrm{GPa}$. It was mainly manifested by the appearance of two diffraction lines at about 2.500 and $1.675 \AA$. Upon decompression, this phase disappeared at about $32 \mathrm{GPa}$.

The ten observed diffraction lines could be indexed with a previously unknown, orthorhombic cell labeled as orthorhombic-III. In order to retain the same general motif for the $\mathrm{ZrO}_{2}$ structure, as in the lower pressure phase, it was necessary to double the $c$ axis (note, that the oxygen sublattice is not visible in our $x$-ray experiment). The search for simplest relations between the unit cells of orthorhombic-I and -III phases gave the following results: $\mathbf{a}_{3}=(1 / 2) \mathbf{a}_{1}+\mathbf{c}_{1}, \quad \mathbf{b}_{3}=\mathbf{b}_{1}, \quad \mathbf{c}_{3}=(1 / 2) \mathbf{a}_{1}-\mathbf{c}_{1}$. The orthorhombic-III phase is achieved by a simple rotation of the unit cell about the $b$ axis (thus, different from that necessary for obtaining the orthorhombic-II phase). The number of formula units is conserved $(Z=8)$ and the change of volume in the transition is about $7.7 \%$. The simulation of the powder diagram within this model gave very satisfactory agreement of both calculated and observed intensities of diffraction lines.

This orthorhombic lattice also accounted for at least three of the four different lines observed in Ref. 18, where a tetragonal symmetry was assumed for this phase (the fourth line is close to a diffraction line from the gasket and was not considered here). From these four lines, ${ }^{18}$ it was not possible to calculate the lattice parameters of the orthorhombic unit cell proposed here. Thus, the volume of the previous tetragonal cell at normal pressure, where we could not retain this orthorhombic cell in a metastable state, could not be used for calculation of the volume variation with pressure. As a result the evaluation of the bulk modulus for the orthorhombic-III phase was not possible.

It should be pointed out that the general structural motif of $\mathrm{ZrO}_{2}$ can be retained in all the high-pressure phases and thus, that the coordination number retains its original value of 7 . The increase of the coordination number proposed in the literature ${ }^{7}$ from 7 to 8 and 9 has to be rejected as it was based on incorrect structures for the orthorhombic-I and orthorhombic-II phases.

The determination of the structures of post-baddeleyite phases is not as straightforward as previously anticipated. From the well-known structure map of $A X_{2}$ compounds it was thought that such phases would have fluorite- or cotunnite-type structures. ${ }^{55}$ Now it is revealed that the cotunnite structure is not found, at least at room temperature up to pressures of $50 \mathrm{GPa}$ and that many different structures can actually by observed: an orthorhombic cell in $\mathrm{ZrO}_{2}$ with $Z=8$, other orthorhombic and tetragonal cells with $Z=4$ in $\mathrm{HfO}_{2}$. This points out the necessi- 
ty for further more detailed studies to be performed in situ.

\section{CONCLUSION}

$\mathrm{X}$-ray in situ powder-diffraction studies have been performed at room temperature on pure zirconia in a diamond-anvil cell using the angular-dispersive method. Under increasing pressure up to about $50 \mathrm{GPa}$, four phases were successively encountered: the ambientpressure monoclinic phase (baddeleyite) and three orthorhombic phases. The unit cells of the two higherpressure phases did not correspond to those previously reported: Neither the orthorhombic, cotunnite $\mathrm{PbCl}_{2}$ type structure nor the tetragonal phase were confirmed. The cotunnite structure previously proposed, mainly by referring to the structure map of $A X_{2}$ compounds, had to be rejected as one forbidden line was distinctly observed under pressure and one other characteristic intense line had not been reported in the earlier studies. The previously reported tetragonal structure, based on the analysis of only four diffraction lines, was also rejected; we could observe many additional lines and thus an orthorhombic cell was proposed. Although the diffraction patterns were of much higher quality in our experiment than previously reported, the correct space group of orthorhombic-II and -III phases in $\mathrm{ZrO}_{2}$ could not be determined. Nevertheless, some structure models might be proposed from the observed relations between the unit cells of the different phases. The structural motif of $\mathrm{ZrO}_{2}$ can be conserved through all the crystallographic transitions and thus, there is no evidence for an increase of the coordination number at room temperature up to $50 \mathrm{GPa}$. Further detailed crystal structure analyses are in progress now.
The unit-cell parameters and the volumes of the different phases have been determined over the whole pressure range of investigation. The bulk moduli of the two lower-pressure phases have been calculated. The value corresponding to the baddeleyite phase is much lower than expected from bulk modulus-volume systematics. It showed that this monoclinic phase is possibly unstable as predicted theoretically. The bulk modulus of the orthorhombic-I phase is, on the other hand, in good agreement with those systematics.

Zirconia was always considered to be analogous to hafnia, $\mathrm{HfO}_{2}$ as they both display the same structural and chemical properties at ambient pressure, and undergo a lower-pressure phase transition to the same orthorhombic structure. However, it is now clear that the phase diagram of hafnia is different from that of zirconia: The phases above $20 \mathrm{GPa}$ are not the same for both compounds (orthorhombic phase III and tetragonal phase IV for hafnia and orthorhombic-II and -III phases for zirconia). The study of quenched samples to compare hafnia and zirconia is of dubious use as the back transformations do not proceed in the same way.

\section{ACKNOWLEDGMENTS}

One of us (A.S.P.) would like to thank the CAPES, Brazilian Government Agency for financial support for the stay in France. P.E.T. would like to thank the French Ministry of Research and Technology for financial support. Both of us would like to thank LPCM for kind hospitality during our stay in the laboratory at the Bellevue campus. Laboratoire de Physico-Chimie des Matériaux is Unité Propre du CNRS.
* On leave from Institute of Low Temperature and Structure Research, Polish Academy of Sciences, 50-950 Wroclaw, Poland.

tOn leave from Instituto de Fisica e Escola de Engenharia da UFRGS, 90035-190 Porto Alegre, R.S. Brazil.

${ }^{1}$ F. W. Vahldiek, L. B. Robinson, and C. T. Lynch, Science 142, 1059 (1963).

${ }^{2}$ E. D. Whitney, J. Electrochem. Soc. 112, 91 (1965).

${ }^{3}$ X. A. Bendeliani, S. V. Popova, and L. F. Vereshchagin, Geokhimiya 6, 677 (1967).

${ }^{4}$ G. L. Kulcinski, J. Am. Ceram. Soc. 51, 582 (1968).

${ }^{5} \mathrm{G}$. Bocquillon and C. Susse, Rev. Int. Hautes Temper. Refract. 6, 263 (1969).

${ }^{6}$ L. M. Lityagina, S. S. Kabalkina, T. A. Pashkina, and A. I. Khozyainov, Fiz. Tverd. Tela (Leningrad) 20, 3475 (1978) [Sov. Phys. Solid State 20, 2009 (1978)].

${ }^{7}$ L. Liu, J. Phys. Chem. Solids 41, 331 (1980).

${ }^{8}$ H. Arashi and M. Ishigame, Phys. Status Solidi A 71, 313 (1982).

${ }^{9}$ S. Block, J. A. H. Da Jornada, and G. J. Piermarini, J. Am. Ceram. Soc. 68, 497 (1985).

${ }^{10}$ L. C. Ming and M. H. Manghnani, in Solid State Physics Under Pressure, edited by S. Minomura (KTK Science, Tokyo, 1985), p. 135.

${ }^{11}$ Y. Kudoh, H. Takeda, and H. Arashi, Phys. Chem. Minerals
13, 233 (1986).

${ }^{12}$ B. Alyzab, C. A. Perry, and R. P. Ingel, J. Am. Ceram. Soc. 70, 760 (1987).

${ }^{13}$ H. Arashi, O. Shimomura, T. Yagi, S. Akimoto, and Y. Kudoh, Adv. Ceram. 24, 493 (1988).

${ }^{14}$ O. Ohtaka, S. Kume, and E. Ito, J. Am. Ceram. Soc. 71, C448 (1988).

${ }^{15}$ D. B. Marshall, M. R. James, and J. R. Porter, J. Am. Ceram. Soc. 72, 218 (1989).

${ }^{16}$ Y. Kudoh, C. T. Prewitt, and H. Arashi, EOS Trans. Am. Geophys. Union, 70, 491 (1989).

${ }^{17}$ O. Ohtaka, S. Kume, and E. Ito, J. Am. Ceram. Soc. 73, 744 (1990).

${ }^{18}$ H. Arashi, T. Yagi, S. Akimoto, and Y. Kudoh, Phys. Rev. B 41, 4309 (1990).

${ }^{19}$ F. Frey, H. Boysen, and T. Vogt, Acta Crystallogr. B 46, 724 (1990).

${ }^{20}$ O. Ohtaka, T. Yamanaka, S. Kume, N. Hara, H. Asano, and F. Izumi, Proc. Jpn. Acad. 66B, 193 (1990).

${ }^{21}$ D. D.Gulamova and S. N. Novoselova, Zh. Neorg. Khimii 36, 1127 (1991) [in Russian].

${ }^{22}$ O. Ohtaka, T. Yamanaka, S. Kume, E. Ito, and A. Navrotsky, J. Am. Ceram. Soc. 74, 505 (1991).

${ }^{23}$ H. Boysen, F. Frey, and T. Vogt, Acta Crystallogr. B 47, 881 (1991). 
${ }^{24}$ W. W. Barker, F. P. Bailey, and W. Garrett, J. Solid State Commun. 7, 448 (1973).

${ }^{25}$ J. Faber, Jr., M. H. Moeller, and B. R. Cooper, Phys. Rev. B 17, 4884 (1978).

${ }^{26}$ P. Aldebert and J.-P. Traverse, J. Am. Ceram. Soc. 68, 34 (1985).

${ }^{27}$ C. J. Howard, R. J. Hill, and B. E. Reichert, Acta Crystallogr. B 44, 116 (1988).

${ }^{28}$ R. N. Patil and E. C. Subbarao, Acta Crystallogr. A 26, 535 (1970).

${ }^{29}$ K. Doi, Bull. Soc. Franc. Miner. Crist. 89, 216 (1966).

30J. Livage, K. Doi, and C. Maziere, J. Am. Ceram. Soc. 51, 349 (1968).

${ }^{31}$ D. K. Smith and H. W. Newkirk, Acta Crystallogr. 18, 983 (1965).

${ }^{32}$ R. Suyama, H. Horiuchi, and S. Kume, Yogyo Kyokai-Shi 95, 567 (1987) [in English].

${ }^{33}$ C. J. Howard, E. H. Kisi, and O. Ohtaka, J. Am. Ceram. Soc. 74, 2321 (1991).

${ }^{34}$ E. H. Kisi, C. J. Howard, and R. J. Hill, J. Am. Ceram. Soc. 72, 1757 (1989).

${ }^{35}$ C. J. Howard, E. H. Kisi, R. P. Roberts, and R. J. Hill, J. Am. Ceram. Soc. 73, 2828 (1990).

${ }^{36}$ S. R. U. Devi, L. C. Ming, and M. H. Manghnani, J. Am. Ceram. Soc. 70, C218 (1987).

${ }^{37}$ L. K. Lenz and A. H. Heuer, J. Am. Ceram. Soc. 65, C192 (1982).

${ }^{38}$ A. H. Heuer and L. H. Schoenlein, J. Mater. Sci. 20, 3421 (1985).

${ }^{39}$ B. C. Muddle and R. H. J. Hannink, Adv. Ceram. 24, 89 (1988).

${ }^{40}$ T. A. Bielecki, Adv. Ceram. 24, 485 (1988).
${ }^{41}$ A. H. Keuer, V. Lanteri, S. C. Former, R. Chaim, R. R. Lee, B. W. Kibbel, and R. M. Dickerson, J. Mater. Sci. 24, 124 (1989).

${ }^{42}$ I. Vedel, A. M. Redon, J. Rossat-Mignod, O. Vogt, and J.-M. Leger, J. Phys. C 19, 6297 (1986).

${ }^{43}$ DICVOL is a program for indexing powder diagrams; D. Louer and M. Louer, J. Appl. Cryst. 5, 271 (1972).

${ }^{44}$ F. Birch, Phys. Rev. 71, 809 (1947).

${ }^{45}$ L. Liu, High Temp. High Pressures 13, 387 (1981).

${ }^{46}$ C. F. Smith and W. B. Crandall, J. Am. Ceram. Soc. 47, 624 (1964).

${ }^{47}$ S. Chan, Y. Fang, M. G. Grimsditch, M. V. Nevitt, W. M. Robertson, and E. Zouboulis, J. Am. Ceram. Soc. 74, 1742 (1991).

${ }^{48}$ T. Mashimo, K. Nagayama, and S. Sawaoka, Phys. Chem. Minerals 9, 237 (1983).

${ }^{49} \mathrm{O}$. L. Anderson, in The Nature of the Solid Earth, edited by $\mathrm{E}$. C. Robertson (McGraw Hill, New York, 1972), p. 575.

${ }^{50}$ R. M. Hazen and L. W. Finger, in Comparative Crystal Chemistry (Wiley, New York, 1982).

${ }^{51}$ R. E. Cohen, M. J. Mehl, and L. L. Boyer, Physica B 150, 1 (1988).

${ }^{52}$ S. Kawasaki, T. Yamanaka, S. Kume, and T. Ashida, Solid State Commun. 76, 525 (1990).

${ }_{53}^{5}$ J.-M. Leger, A. Atouf, P. E. Tomaszewski, and A. A. Pereira, Phys. Rev. B (to be published).

${ }^{54}$ L. Liu, in High Pressure in Geophysics, edited by S. Akimoto and M. H. Manghnani (Center Academic, Tokyo, 1982), p. 349.

${ }^{55}$ K. F. Seifert, Fortschr. Mineral. 45, 214 (1968).

${ }^{56}$ R. Suyama, T. Ashida, and S. Kumi, J. Am. Ceram. Soc. 68, C237 (1985). 